\title{
ANAESTHETIC MANAGEMENT OF A PATIENT WITH WILSON'S DISEASE POSTED FOR EMERGENCY LSCS: A CASE REPORT
}

Kousalya Chakravarthy', Srinivas Reddy Nalagandla², Sunil Kumar Cherukuri3, Radha Ramana Murthy Konda4

\section{HOW TO CITE THIS ARTICLE:}

Kousalya Chakravarthy, Srinivas Reddy Nalagandla, Sunil Kumar Cherukuri, Radha Ramana Murthy Konda. "Anaesthetic Management of a Patient with Wilson's Disease Posted for Emergency LSCS: A Case Report". Journal of Evolution of Medical and Dental Sciences 2015; Vol. 4, Issue 47, June 11; Page: 8229-8233, DOI: $10.14260 /$ jemds/2015/1192

ABSTRACT: Wilson's disease (WD), or hepatolenticular degeneration, is a rare autosomal recessive disorder with a prevalence of 1:50,000-1:100,000 live births. ${ }^{1}$ The genetic disorder causes a reduction in the synthesis of the copper transporting protein ceruloplasmin. Clinical presentation may vary from asymptomatic patients to those presenting with fulminant liver disease or neurological symptoms or psychiatric illness. The course of events in a pregnant patient depends upon the preconception status of the disease spectrum. The neuro-psychiatric illness and hepatorenal involvement in antenatal period may get worsened. The risk of ascitis, Gatrointestinal bleeding and Varicial rupture is increased in pregnant women with pre-existing cirrhosis. Elevated serum copper levels are associated with post-partum depression (PPD). Copper accumulation may lead to erythrocyte hemolysis resulting in Coombs negative haemolytic anaemia (10-15\%). There is also an increased risk of Hypertensive disorders in Pregnancy, HELLP syndrome and placental abruption. The disease status at the time of presentation for surgery should determine the mode of Anaesthesia, intraoperative management and post-operative care. Hepatic dysfunction can cause delayed recovery with General anaesthesia. Pre-existing neuro psychological problems may get aggravated by the hypnotic and sedative drugs. Excessive copper may interfere with neuromuscular transmission. Pre existing neurological deficiency must be documented before attempting an Epidural or a subarachnoid block. We report a case of a primigravida with previously diagnosed Wilson's disease initially presented with neurological symptoms and muscular weakness in the lower limbs. Though treated for 3yrs with trientene and zinc, she was on irregular treatment when posted for Emergency Caesarean section. After a proper Preanesthetic evaluation of the patient, LSCS was conducted under subarachnoid block. A good intra operative and post-operative monitoring resulted in good maternal and neonatal outcome. We conclude that regional anaesthesia can be safely administered for Caesarean delivery in patients with Wilson's disease.

KEYWORDS: Wilson's disease; Trientine; Pregnancy; Caesarean Section, Subarachnoid block.

INTRODUCTION: Wilson's disease (WD) is a rare autosomal recessive disorder with a prevalence of 1:50,000-1:100,000 live births. ${ }^{1}$ The molecular pathogenesis is mutation in the P-type ATPase ATP7B gene, which is highly expressed in the liver, kidney, and placenta. ATP7B has two main intracellular roles within the liver; promoting incorporation of copper into apoceruloplasmin and excretion of excess copper into the bile. ${ }^{2}$ Patients may present with hepatic and neurological features, extrapyramidal symptoms and Kayser-Fleischer corneal rings. Neurological manifestations include tremors, dysarthria, rigid dystonia, seizures, and psychiatric disorders. Liver involvement can lead to acute liver failure, chronic hepatitis or cirrhosis. The involvement of Liver and Nervous system makes both General and regional anaesthetic techniques a challenge to the anaesthesiologist. In the present 
case we discuss the anaesthetic management of a pregnant patient with Wilson's disease posted for emergency LSCS successfully managed by regional anaesthesia.

CASE REPORT: A 19 yrs old unbooked primigravida, a known case of Wilson's disease, at 37 wks of gestation was admitted in early labour. In view of severe oligohydramnios and presumed fetal compromise, patient was posted for Emergency LSCS - Category 2.

History taken during Pre Anesthetic Evaluation revealed that the patient presented with neurological symptoms 3 yrs back at the age of $16 \mathrm{yrs}$, in the form of waddling gait, progressive difficulty in walking, difficulty in standing. She was diagnosed to have myopathy with proximal muscle weakness, polyarthralgia and contracture at right elbow joint. Elevated level of copper in the urine $-325 \mu \mathrm{g} /$ day (Normal $<60 \mu \mathrm{g} /$ day) and low serum ceruloplasmin level of $0.1 \mathrm{~g} / \mathrm{l}$; (Normal range $0.25-0.63 \mathrm{~g} / \mathrm{l}$ ), Kayser-Fleischer corneal rings and MRI signal morphology and distribution of lesions in favour of Wilson's disease, clinched the diagnosis of WD. In view of the neurological symptoms patient was initially stabilized with combination of Carbidopa $25 \mathrm{mg}$ and Levodopa $100 \mathrm{mg}$ (Tab. Syndopa plus $1 / 2$ bid) and started on Tab. Trientene and zinc. There was symptomatic improvement. Patient stopped taking the medications since $5^{\text {th }}$ month of gestation.

None of the family members suffered with Wilson's disease. There was no history of jaundice or features suggestive of hepatic dysfunction in the past. No history of epilepsy.

On examination the patient was moderately built with a BMI of $22 \mathrm{Kg} / \mathrm{m}^{2}$, there was no pallor, no icterus, no cyanosis, no clubbing, pupils-NSRL, DTR were brisk. Vitals: Patient was afebrile, pulse84beats/min, regular, $\mathrm{SPO}_{2}$ 98\% on room air, B.P:120/80mmHg. Cardiovascular and respiratory system were WNL; there were no residual neurological signs in the lower limbs. Motor power was normal 5/5 in all the four limbs. Airway and spine were normal.

\begin{tabular}{|c|c|}
\hline 1 & Hemoglobin: $13.8 \mathrm{~g} / \mathrm{dl}$ \\
\hline 2 & Platelet count: 1.7 lakh cells / $\mathrm{mm}^{3}$ \\
\hline 3 & Blood grouping, Rh typing: A Positive \\
\hline 4 & KFT: S.Creatinine : $0.9 \mathrm{mg} / \mathrm{dl} ; \mathrm{Na}^{+}: 142 \mathrm{mmol} / \mathrm{L} \mathrm{K}+: 3.7 \mathrm{meq} / \mathrm{L}, \mathrm{Cl}-99 \mathrm{meq} / \mathrm{L}$ \\
\hline 5 & $\begin{array}{l}\text { LFT: serum alanine aminotransferase (ALT): } 24 \mathrm{IU} / \mathrm{L} \text {, the serum } \\
\text { aspartate aminotransferase (AST): } 38 \mathrm{IU} / \mathrm{L} \text {, Bilurubin : } 0.8 \mathrm{mg} \%\end{array}$ \\
\hline 6 & PPBS: $118 \mathrm{mg} \%$ \\
\hline 7 & Thyroid Profile: WNL \\
\hline 8 & APTT: 32, PT 13 sec; INR : 1.06 \\
\hline 9 & Serology: HbSAg, HIV negative \\
\hline 10 & USG fetus: single live foetus in cephalic presentation. Placenta upper and posterio \\
\hline
\end{tabular}

NBM status of 6hrs confirmed. Antacid prophylaxis was given in the form of IV Ranitidine 50mg and IV Ondensetron 4mg. Intraoperative monitoring was done with Pulse oxymeter, NIBP, 3 Lead ECG and Urine output. Co- hydration was done with $500 \mathrm{ml}$ of warmed Ringer Lactate. Single shot subarachnoid block was given in Right lateral position with 26G Quinke needle. 10mg 0.5\%Bupivacaine with $20 \mathrm{ug}$ of Fentanyl injected into the Sub arachnoid space. Sensory block up to T4 level confirmed. LSCS was uneventful. A $2.9 \mathrm{Kg}$ male child was delivered with APGAR 8, 9, 9. There were no obvious congenital malformations in the new born. Blood loss was approximately $300 \mathrm{ml}$. $2 \mathrm{~L}$ of crystalloids were given in the intraoperative period. Patient was shifted to POW in haemo- 


\section{CASE REPORT}

dynamically stable condition. Postoperative period was uneventful. Regression of the block was noticed 2hours after the procedure. There was no neurological damage post subarachnoid block. Patient was ambulated 8hrs after surgery. Postoperative analgesia was maintained with a combination of Fentanyl, Diclofenac and Paracetamol. The patient was carefully monitored for any psychological depression. Both mother and child were discharged on $5^{\text {th }}$ POD in stable condition with advice to resume Neurologist consultation for further management.

DISCUSSION: The course of events in a pregnant patient depends upon the preconception status of the disease spectrum. The excessive accumulation of copper can lead to infertility and effect embryogenesis leading to spontaneous, threatened and missed abortions or blighted ovum. Early recognition and effective treatment can ameliorate the undesirable effects of copper accumulation in Wilson's disease. Medical therapy includes reducing dietary copper intake, antagonizing its absorption with zinc, or chelation with penicillamine or trientine. All the three has equal efficacy \& safety during pregnancy.3,4 The treatment should be commenced before conception and continued during pregnancy.

EFFECT OF WILSON'S DISEASE ON PREGNANCY: Effect of Wilson's disease on pregnancy is essentially due to either accumulation of copper or due to much lowered plasma copper levels because of medication used for chelating Copper. Excessive copper is teratogenic and has been associated with fetal intrauterine growth restriction and neurological sequelae. Elevated serum copper levels are associated with post-partum depression (PPD). There is also an increased risk of Hypertensive disorders in Pregnancy, HELLP and placental abruption associated with Wilson's disease 5,6 .

EFFECT OF PREGNANCY ON WILSON'S DISEASE: The physiological changes in pregnancy causes an increase in the of serum-ceruloplasmin level. This along with the increased copper requirements of the fetus may lead to decreased maternal serum copper levels. Both these factors may contribute to the neutral stance of the disease process during pregnancy. However, decreased copper levels alone are not sufficient for an uneventful pregnancy outcome. Though pregnancy does not aggravate the disease process, the presence of neuro-psychiatric illness and hepatorenal involvement in antenatal period may get worsened. The risk of ascitis, Gatrointestinal bleeding and Varicial rupture is increase in pregnant women with pre-existing cirrhosis.

EFFECT OF ANAESTHESIA ON WILSON'S DISEASE: There are very few reports in literature on anaesthesia for Wilson's disease. Regional, General and also plexus blocks have been described. Hobaika et al described successful interscalene brachial plexus block in a patient with Wilson's disease presented with Osteomuscular symptoms. ${ }^{7}$ Successful General anaesthesia has been described by Baykal et al in a 4yr old child with Wilson's disease. ${ }^{8}$ Hepatic dysfunction can cause delayed recovery with General anaesthesia. ${ }^{9}$ Copper accumulation may lead to erythrocyte hemolysis resulting in Coombs negative haemolytic anaemia (10-15\%).10 Pre-existing neuro psychological problems may get aggravated by the hypnotic and sedative drugs. Excessive copper may interfere with neuromuscular transmission. ${ }^{10}$ Decreased pseudocholinesterase levels slow the metabolism of succinyl choline. This may increase the risks of airway management, RSI (Rapid Sequence Induction) for General anaesthesia in Obstetric population. Decreased hepatic metabolism, decreased renal excretion, altered neuromuscular transmission, decreased muscle power may all lead to increased sensitivity of the patients to ND NMBDs (Nondepolarizing neuromuscular blocking drugs). 
The presence of associated co morbid conditions in pregnancy may pose a relative contraindication to Central Neuraxial Blockade, as in Pre eclampsia with HELLP syndrome, Thrombocytopenia or Altered coagulation profile. An INR $<1.4$ or platelets $>100,000 \mathrm{~mm}^{-3}$ is acceptable for a CNB. ${ }^{10,11}$ Pre-existing neurological deficiency must be documented before attempting an Epidural or a subarachnoid block. The disease status at the time of presentation for surgery should determine the mode of Anaesthesia, intraoperative management and post-operative care.

In our case the patient initially presented with neurological symptoms and muscular weakness in the lower limbs. Though treated for 3yrs with trientene and zinc, at the time of surgery, she was not on medication. The presentation of the patient for an emergency surgery did not allow enough time for thorough evaluation. A complete regression of the symptoms, good sensory and motor power in all the limbs and absence of detectable residual neuromuscular weakness pointed towards a period of remission of the disease. Hence we opted for a subarachnoid block.

CONCLUSION: We conclude that regional anaesthesia can safely be administered in asymptomatic pregnant patients with Wilson's disease. A thorough preanaesthetic evaluation should determine the status of the disease to rule out any contraindications to regional anaesthesia. Meticulous preoperative optimization and planning of anaesthesia, intra operative and postoperative care can lead to a successful materno- fetal outcome in Wilson's disease.

\section{REFERENCES:}

1. Ayesha Malik, Ali Khawaja, Lumaan Sheikh Malik. Wilson's disease in pregnancy. Case series and review of literature BMC Research Notes 2013; 6: 421.

2. E. A. Roberts, B. Sarkar. "Liver as a key organ in the supply, storage, and excretion of copper." American Journal of Clinical Nutrition 2008; 88: 581S-84S.

3. Sanjoy kumar bhattacharyya, Shyama prasad saha, Jaydeep samanta. Pregnancy in a woman with wilson's disease Treated with zinc: a case report. Korean J Obstet Gynecol 2012; 55(3): 180-82.

4. Roberts EA, Schilsky ML. American Association for Study of Liver Diseases (AASLD). Diagnosis and treatment of Wilson disease: an update. Hepatology 2008; 47: 2089-111.

5. Theodoridis TD, Zepiridis L, Athanatos D, Dinas K, Tzevelekis F, Bontis JN. Placenta abruption in a woman with Wilson's disease: a case report. Cases J 2009; 2: 8699.

6. Członkowska A, Gromadzka G, Büttner J, Chabik G. Clinical features of hemolysis, elevated liver enzymes, and low platelet count syndrome in undiagnosed Wilson disease: report of two cases. Arch Gynecol Obstet 2010; 281: 129-34.

7. A. B. De Souza Hobaika. "Anesthesia for a patient with Wilson's disease-a case report." Middle East Journal of Anesthesiology 2008; 4: 905-8.

8. Mehmet Baykala, Sami Karapolatb. Anesthetic Management of a Pediatric Patient With Wilson's Disease. J Clin Med Res 2010; 2(2): 99-101.

9. Maze M, Bass NM. Anesthesia and the hepatobiliary system In: Miller RD, Editor. Miller's Anesthesia. 5th Ed. Philadelphia, Churchill Livingstone; 2000, p1960 - 72.

10. Adrian Langley, Charles T Dameron. Review Article Copper and Anesthesia: Clinical Relevance and Management of Copper Related Disorders. Anesthesiology Research and Practice Volume 2013. 


\section{CASE REPORT}

11. Sujata Chaudhary and Rashmi Salhotra. Subarachnoid block for caesarean section in severe pre eclampsia. J Anaesthesiol Clin Pharmacol 2011; 27(2): 169-73.

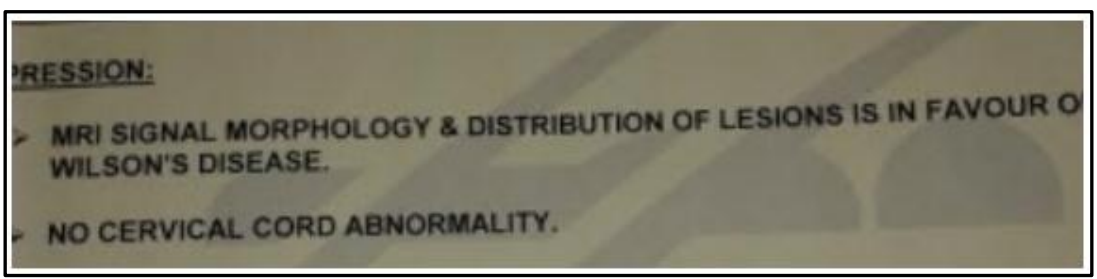

MRI report confirming Wilsons Disease

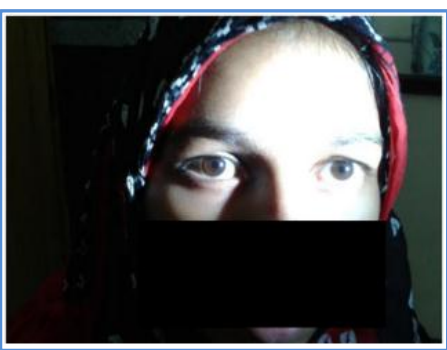

\section{KF Ring in both eyes}

\section{AUTHORS:}

1. Kousalya Chakravarthy

2. Srinivas Reddy Nalagandla

3. Sunil Kumar Cherukuri

4. Radha Ramana Murthy Konda

\section{PARTICULARS OF CONTRIBUTORS:}

1. Assistant Professor, Department of Anaesthesia, Niloufer Hospital for Women and Children, Hyderabad.

2. Assistant Professor, Department of Anaesthesia, Niloufer Hospital for Women and Children, Hyderabad.

3. Assistant Professor, Department of Anaesthesia, Niloufer Hospital for Women and Children, Hyderabad.

\section{FINANCIAL OR OTHER}

COMPETING INTERESTS: None
4. Professor \& Chief Anesthesiologist, Department of Anaesthesia, Niloufer Hospital for Women and Children, Hyderabad.

\section{NAME ADDRESS EMAIL ID OF THE CORRESPONDING AUTHOR:}

Dr. Kousalya Chakravarthy, Flat 504, C-Block, Amsri Central Court, Old Lancer Lines, Secunderabad-500025.

E-mail: dr.kausi.c@gmail.com

Date of Submission: 20/05/2015. Date of Peer Review: 21/05/2015. Date of Acceptance: 04/06/2015. Date of Publishing: 11/06/2015. 